\title{
The Design of Multi-protocol Converter Based on Functional Analysis
}

\author{
Yan Wu, Jingjing Zhou \\ School of of information engineering JiangXi University of Technology, NanChang, 330098, China
}

\begin{abstract}
A multi-protocol converter was developed by embedded system, which used a method of protocol analysis based on character set to establish both a sequence of command instructions and a sequence of protocol analysis methods for one serial port, used an analysis method of character set to collect and extract the data independently. The command sequence and protocol analysis methods could be selected and configured to meet varieties of analysis and conversion in different applications.
\end{abstract}

Keywords-protocol converter; configurable; characteristics of characters; sequence

\section{INTRODUCTION}

The operation of interconnection between intelligent devices whose key issue is communication can be seen everywhere.[1] Usually, the data exchange must be executed with the same protocol by communicated parties. Unfortunately, in practice, even the communication terminals from different manufacturers with the same function have different protocols. It will inevitably cause inconvenience to communication. Therefore, the protocol conversion is considered as the optimal program to achieve interoperability for a long period. Such as literature [3] has proposed a program which uses a protocol converter for different intelligent devices in accordance with relevant standards in telecommunications system. The program applies to the telecommunications industry and terminals which provide a standard. Literature [4] has developed a communication protocol converter which has two serial ports, when one serial port has received a frame of data, the other one sends the data in a new frame format, but its software is different depends on different communication protocols. Literature [5] has invented a system of communication protocol conversion, in which the frame of serial data stream could be separated even though it does not have specific definition of character and its size is unknown. Literature [6] has designed a protocol converter by SCM (Single-chip Microcomputer), which is self-collecting peripheral data in manner of polling. In order to avoid traffic congestion, one peripheral is permitted to communicate with the master at the same time. Literature [7] has proposed a protocol converter, which can convert various protocols with multi-interface. Literature [8] has raised a communication protocol converter by ARM (Advanced RISC Machines) which can achieve the conversion between the common protocols and custom protocols. It also can cross conversion and forward various protocols in power system. Thus, the conventional application method of protocol conversion is shown in
Fig.1, which adds a protocol converter between the peripheral and master device. It only realizes the functions of matching the communication parameters of serial port, converting the format, forwarding the data. Therefore, the urgent problem of protocol converter is how to adapt different devices, how to collect and extract the data autonomously, and how to reduce the complexity of control for practical application.

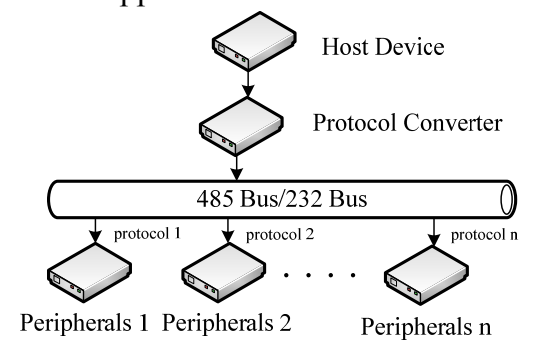

Figure 1. System block diagram

\section{HARdware Design of Protocol CONVERTER}

The hardware schematic of protocol converter is shown in Fig.2, which is composed mainly by LPC2368 whose core is ARM7, external watchdog, memory and communication modules of serial port.

This protocol converter uses LPC2368 as the main chip. It is rich in internal resources and supporting small-scale operating systems, such as $\mu \mathrm{C}$-OS II. There are four serial ports, I2C bus and RAM, which can effectively simplify the peripheral hardware circuit. In the figure, the external watchdog will reset ARM when it is abnormal. The configuration parameters (such as baud rate, data bits, stop bits, and parity bit), command sequence and analysis method are all stored in EEPROM (Electrically Erasable Programmable Read-Only Memory). One of four serial ports connects to master device fixedly (The configuration of serial port and protocol are fixed), and the other serial ports connect to peripherals. 


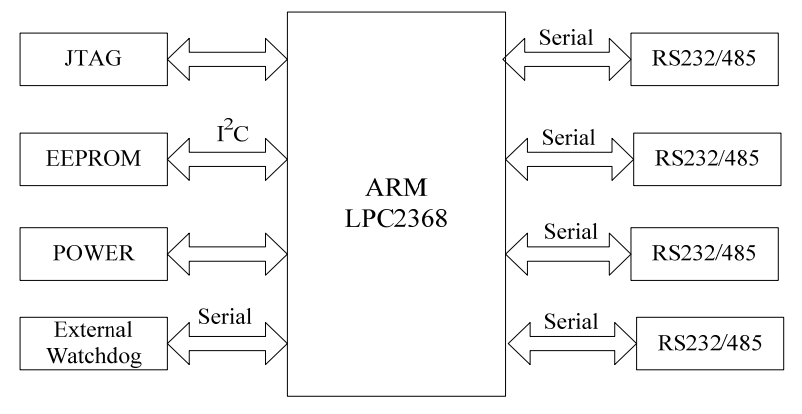

Figure 2. Hardware block diagram

\section{SOFtware OF Protocol CONVERTER}

\section{A. Interaction model to peripheral}

Protocol converter lies in software. Take a serial port for example to show how to communicate with peripherals, shown in Fig.3, it mainly includes configuration of communication parameters, command sequence, received sequence of peripheral data, analysis methods and data packed. Communication parameters of serial port mainly includes baud rate, data bits, parity bit and stop bit, so as to match the different configuration of peripheral serial port. Command sequence is responsible for sending the corresponding command to peripheral by serial port. The format of commands is: the content in first address means the length of command, and the after address saves the home address of command. Analysis method is rules that how to process the received data. All of these parts in Fig. 3 are stored in EEPROM and can be modified. Protocol converter sends the command to peripherals with timing and turning. The data is analyzed and packed after completely being received.

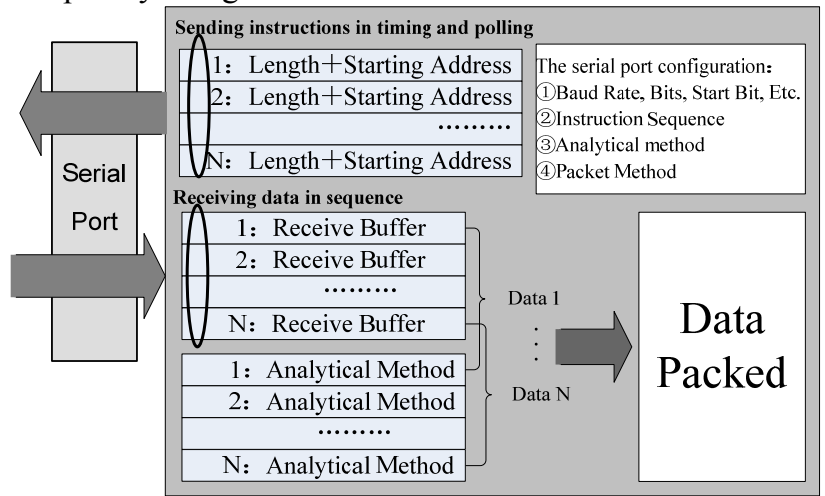

Figure 3. The interaction model to peripheral

\section{B. The command sequence and polling of peripherals}

Usually, in practical application, if the data can be obtained in auto polling, it will help to improve the application effect of protocol converter undoubtedly. The protocol converter sends multi-command with timing and turning to query peripheral by one serial port. The manner of it includes command sequence and timer. The timing function of timer can be achieved by timer interrupt of embedded system. In the regular polling, the protocol converter sends a command with being pointed by the pointer to peripheral, then the pointer moves back to point to the next command. If the current command is null (usually, the length of contents is zero), then the pointer moves back until it finds a valid command. If the pointer is pointing to the tail of the command, the pointer will move back to the first command buffer.

\section{Receive peripheral data in sequence}

Usually, the communication of serial port to peripherals is continuous, so a packet of data is continuously sent or received byte by byte. The waiting time of sending or receiving data between one byte and the next byte must be equal to sending or receiving a byte data (the specific time relates to the baud rate), taking the baud rate is 9600 for example, the time of sending or receiving a byte data is about $0.84 \mathrm{~ms}$, so the waiting time of sending or receiving data between one byte and the next byte is about $0.84 \mathrm{~ms}$. In practical application, the time interval between two packets of data is generally much more than sending or receiving a byte data. Therefore, the adjacent time span of receiving data between two bytes can be used as a basis to judge whether the continuous communication of serial port is ending. This design sets the time interval is $200 \mathrm{~ms}$ to determine whether a data reception is complete. The protocol converter receives the peripheral data in sequence, and the model of it is shown in Fig.4. The data receiving is by way of switching the receive buffer in rotation, first initializing the two pointers about Head and Tail of buffer array pointing to the same buffer, and defining the buffer which is pointed to by Tail as the receive buffer. Then as long as the receive buffer completed receiving data, the Tail pointer will move back to point to the next buffer. At the same time, as long as the Head and Tail pointer does not point to the same buffer, extracting the data of buffer which the Head pointer is pointing to process, and then Head pointer moves to point to the next buffer. Once the Head pointer is equal to Tail pointer, shows that all the data have been processed or no data is received.

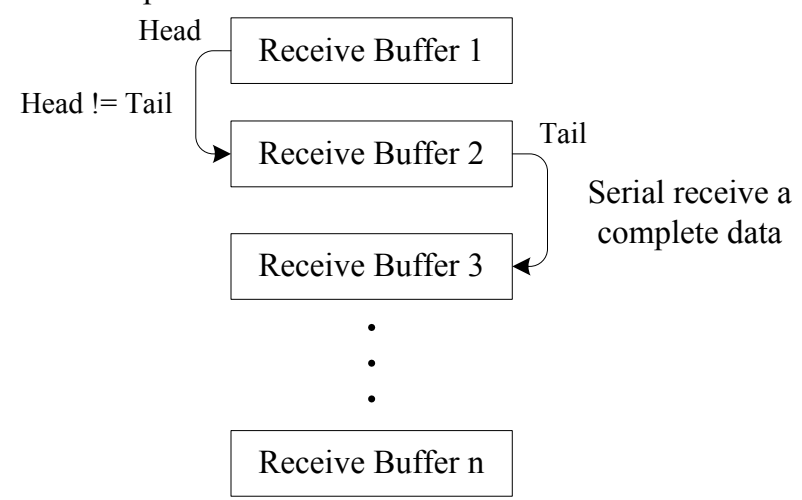

Figure 4. The model of receiving peripheral data in sequence 


\section{The analysis of data stream based on characteristic of characters set}

Usually, when terminal equipment is asked, it will return large amounts of data, but users often only need to know some of the main data, so it is necessary to filter out the data (the existing protocol converter is basically not to deal with the data, but to return all of it).

In order to analyze and intercept data, the protocol converter uses a method of analyzing data stream based on characteristic of characters set. It is composed of characteristic of characters set, protocol rules, analysis data and analysis. The characteristic of characters set is consisted of the character rule which may arise, it can be modified. The composition of the protocol rule includes characteristic of characters, starting address and description of length, shown in Fig.5. The characteristic of characters are composed of all characters except numbers, on behalf of the meaning of intercepted data. The starting address represents physical address of intercepted data, and the description of length represents the length of data. The type of starting address and description of length are fixed as unsigned integer, its size range is from 0 to 255 . Analysis data is the received data of peripheral which is stored in receive buffer, analyzed through the protocol rule, and then parsed out. The analysis part searches characteristic of characters set by protocol rule and uses the starting address to obtain the physical address of each character byte in data stream which is described by characteristics of characters in protocol, and gets the data of specific length through description of length.

The process of data analysis based on characteristic of characters set is shown in Fig.6, first of all, analyzing the description of data flow and generalizing the characteristic of characters set, second, analyzing data protocols and setting the protocol rules corresponding to peripheral, and then after waiting to receive a completed data, reading a bunch of characteristic of characters through characteristic of characters set in accordance with the separator character and searching characteristic of characters in the string of protocol rule, after retrieving characteristic of characters in protocol rule, getting the starting address and description of length, thus intercepting the specified data in analysis data. For example: If the protocol rules is the "A001004", the received data is "1234567890", that $\mathrm{A}$ is the characteristic of character, 001 is the starting address, 004 is the length. So the "A001004" extracts the data is "1234".

\begin{tabular}{|c|c|c|}
\hline $\begin{array}{c}\text { Characteristics of } \\
\text { Characters }\end{array}$ & Starting Address & $\begin{array}{c}\text { Description of } \\
\text { Length }\end{array}$ \\
\hline
\end{tabular}

Figure 5. The model of protocol rule

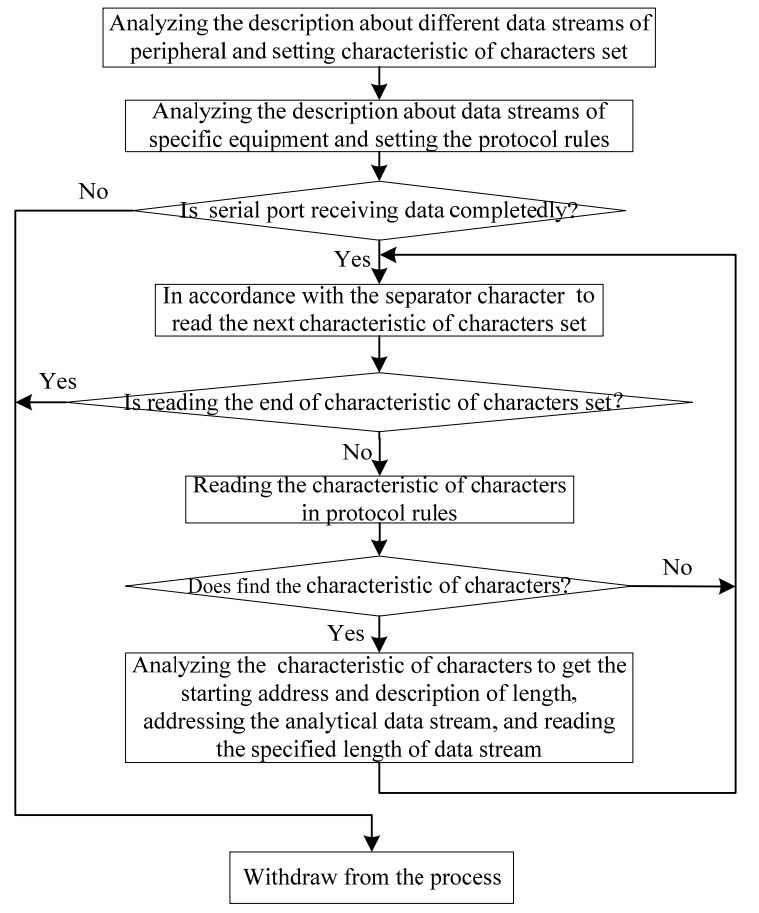

Figure 6. The process of data analysis based on characteristic of characters set

\section{APPLICATION AND CONCLUSIONS}

The protocol converter has been used in lighthouse beacon in Hainan province to accomplish the communication from master to various terminal equipments of PLC, radar transponder, light and other devices to achieve the unified management, in which the protocol converter is responsible for the function of matching communication parameters between the master and terminal equipment, extracting effective data, timing check terminal equipment parameters, and so on, it can solve the problem of our customers often require to add or replace different devices effectively. After a long period of using, the protocol converter is stable with good effect.

\section{REFERENCES}

[1] Liu Xiyuan, Shen Guomin, Li Feng, etc. Application of compiling principle to conversion of communication protocols[J]. Journal of Gansu Industrial University, 26(3), pp.69-72,2012.

[2] Liu Jie, Liu Bin, Yang Xueliang, etc The Discussion of Protocol Converter Synthesis Methods[J]. Computer Application,19(4), pp.30-32,2012.

[3] Hao Juan, Ye Zhixiang. Study on Serial Communication Protocol Converter[J]. Computer Development \& Applications, 15(1), pp.37-38, 2011.

[4] Miao Shihong, Wang Shaorong, Liu Pei, etc. Implementation of the Converter of Communication Protocols for Intelligent Devices[J]. Automation Instrumentation, 21(5), pp.27-28,2011.

[5] Chen Wenjian, Xie Jiachun, Xu jun, etc. The Protocol Conversion System of Serial Communication Frame Based on MCU and CPLD[J]. Computer Application, 23(1), pp.222-223,2013. 
[6] Yang Zhenglin, Wang Sihua. Protocol Conversion Instrument Based on Single Chip MCU and the Application in Power Monitoring System[J]. Microcomputer Applications,27(3), pp.346-348,2010

[7] Zhang Ke, Zhang Hongjuan, Design and Implementation of Multi-interface Protocol Converter[J]. Electronic Engineer,35(7), pp.33-35, 2009.
[8] Sang Wei, Wang Shaorong. Design of ARM-Based Communication Protocol Commutator[J]. Electric Engineering,23 (4), pp.44-45, 2011. 